\title{
Abstraction and problem reformulation
}

\author{
Fausto Giunchiglia \\ Mechanized Reasoning Group \\ IRST \\ 38050 Povo, Trento, Italy
}

In work done jointly with Toby Walsh, the author has provided a sound theoretical foundation to the process of reasoning with abstraction [GW90c; GWS9; GW90b; GW90a]. The notion of abstraction formalized in this work can be informally described as:

[property 1 ] the process of mapping a representation of a problem, called (following historical convention [Sac74]) the "ground" representation, onto a new representation, called the "abstract" representation, which:

[property 2 ] helps deal with the problem in the original search space by preserving certain desirable properties and

[property 3 ] is simpler to handle as it is collstructed from the ground representation by "throwing away details".

One desirable property preserved by an abstraction is provability; often there is a relationship between provability in the ground representation and provability in the abstract representation. Another can be deduction or, possibly inconsistency. By "throwing aivay details" we usually mean that the problem is described in a language with a smaller search space (for instance a propositional language or a language without variables) in which formulae of the abstract representation are obtained from the formulae of the ground representation by the use of some terminating rewriting technique. Often we require that the use of abstraction results in more efficient reasoning. However, it might simply increase the number of facts asserted (eg. by allowing, in practice, the exploration of deeper search spaces or by implementing some form of learning).

Among all abstractions, three very important classes have been identified. They relate the set of facts provable in the ground space to those provable in the abstract space. We call:

- TI abstractions all those abstractions where the abstractions of all the provable facts of the ground space are provable in the abstract space;

- TD abstractions all those abstractions where the

\author{
$\therefore$ 'í \\ "unabstractions" of all the provable faets of the ab- \\ stract space are provable in the ground space;
}

$1<+1,6$

- TC abstractions all those abstractions where a fact is provable in the ground space if and only if its abstraction is provable in the abstract space.

Historically the word abstraction has been mainly used with a much more restricted meaning which captures its use in problem solving and planning (for instance in Abstrips or Soar). Our notion of abstraction (and in particular the three classes defined above) turns out to capture and provide and unifying framework for describing work done in the definition of decision procedures (see for instance [DG79; Giu91]), in planning and problem solving (see for instance (Sac73; Ell90; MH91; Ḱno89]), explanation (see for instance [Doy86]), common sense reasoning (see for instance [Hob85]), qualitative and model based reasoning (see for instance [Moz90; Wel91]), approximate reasoning [Imi87]), analogy (see for instance [Ble90]) and reasoning with very large data bases (see for instance [Lev92]).

At a close look abstraction seems also very related to problem reformulation. In particular it seems that problem reformulation can be characterized as using some of the subclasses of TC and TD abstractions introduced in [GWOOC]. A positive feedback on this intuition would allow to use the framework described in [GW90c; GW89] to put the work on problem reformulation on a more solid ground and, at the same time, to study and compare the techniques used in problem reformulation with the techniques used in all the other areas captured by the framework.

\section{References}

IV.W. Bledsoe. A precondition prover for analogy. CS Dept. memo, 1990.

B. Dreben and W.D. Goldfarb. The Decision problem - Solvable classes of quantificational formulas. Addison-Wesley Publishing Company Inc., 1979.

R.J. Doyle. Constructing and refining causal explanations from an inconsistent domain theory. In Proc. 
Fifth National Conference on Artificial Intelligence, Philadelphia, PA, 1986. AAAI.

T. Ellman. Mechanical generation of heuristics through approximation of intractable theories. In Working Notes of AAAI Workshop on Automatic Generation of Approximations and Abstractions, Boston, MA, 1990.

E. Giunchiglia. A set of hierarchically structured decision procedures for some subclasses of First Order Logic. In Proceedings 3rd Scandinavian Conference on Artificial Intelligence, Roskilde University, Denmark, 1991. Also available as MRG-DIST Technical Report 9101-01, University of Genova, Italy.

F. Giunchiglia and T. Walsh. Abstract Theorem Proving. In Proc. IJCAI 89, 1989. IRST Technical Report 8902-03. Also available as DAI Research Paper No 430, University of Edinburgh.

F. Giunchiglia and T. Walsh. Abstraction in AI. AISB Quarterly, 73:22-26, 1990.

F. Giunchiglia and T. Walsh. The inevitability of inconsistent abstract spaces. Technical Report 900 16, IRST, Trento, Italy, 1990. Also available as DA: Research Paper, University of Edinburgh. Accepted to the Journal of Automated Reasoning.

F. Giunchiglia and T. Walsh. A Theory of Abstraction. Research paper no. 516, Dept. of Artificial Intelligence, University of Edinburgh, 1990. Also available as IRST-Technical Report 9001-14. To appear in Artificial Intelligence.

J.R. Hobbs. Granularity. In Proc. 9lh IJCAI conference, pages 432-435. International Joint Conference on Artificial Intelligence, 1985.

T. Imielinski. Domain abstraction and limited rea. soning. In Proc. 10th IJCAI conference, pages 9971003. International Joint Conference on Artificial Intelligence, 1987.

C.A. Knoblock. Learning hierarchies of abstraction spaces. In Proc. Sixth Intnl. Workshop on Mfachine Learning, 1989.

A.Y. Levy. Irrelevance in problem solving. In $S u b$ mitted to $A A A I-92,1992$.

I. Mozetic and C. Holzbaur. Extending EBG by abstraction operators. In Proceedings E WSL-91, Porto, Portugal, 1991. Springer-Verlag.

I. Mozetic. Abstractions in model-based diagnosis. In Working Notes of AAAI-90 Workshop on Aulomatic Generation of Approximations and Abstractions, pages 64-75. AAAI, 1990.

E.D. Sacerdoti. Planning in a Hierarcliy of Abstraction Spaces. In Proc. 3rd IJCAI conference. International Joint Conference on Artificial Intelligence, 1973.

E.D. Sacerdoti. Planning in a hierarchy of abstraction spaces. Artificial Intelligence, 5:115-135, 1974.
D.S. Weld. Reasoning about model accuracy. Technical Report 91-05-02, University of Washington, Dept. Computer Science and Engineering, 1991. 